\title{
Allele Frequencies of 15 Autosomal STR Loci in Hilla City population / Iraq
}

\author{
Halah Kh. Ibrahim Al-Sammarraie Majeed Arsheed Sabbah Mohammed Mahdi AL-Zubaidi \\ Forensic DNA Research and Training Center / Al-Nahrain University \\ E-mail: Hala_kh76@yahoo.com
}

\begin{abstract}
Back ground: It is important to establish STR database for future applications in forensic and paternity tests to gain a reliable evaluation for statistical analysis and that STR database would be more representative.

Objective: The objectives of this study are establishing autosomal STR database for the population of Hilla City.

Material and methods: DNA was extracted and purified from sixty six individuals from Hilla city by Prepfiller kit. Extracted DNA quantified by Quantifiler real time PCR kit. Fifteen autosomal STR loci (Identifiler) were amplified then analyzed by Genetic Analyzer. All kits supplemented by Applied Biosystem, USA.

Results: All samples amplified and analyzed successfully. Allele frequency and forensic parameters were determined.

Conclusion: We can conclude that we can use this database for further forensic and paternity applications.
\end{abstract}

Key words: STR, Forensic DNA, Paternity, Database, Hilla city.

\section{Introduction}

Short tandem repeats (STR) are DNA loci with repeat motifs of (2-8bp) lengths which are highly polymorphic in human population (1). STR is a valuable tool for human typing due to its small size, widespread distribution and their highly in formativeness (2). Many companies such as Applied Biosystem and Promega were manufactured STR kits for typing human populations. These kits used to establish STR databases database in countries and cities, including Iraq, for future use in human identification, paternity and criminal investigation $(3,4,5,6)$. Several parameters were established as a quality control for the STR database such as forensic parameters [matching probability (MP), power of discrimination (PD), polymorphism information content (PIC), power of exclusion (PE)] and paternity parameters such as paternity index (PI) and population genetics parameters such as hardy weinberg equilibrium (HWE) and linkage equilibrium (LE) (7). In Forensic DNA center, Alnahrain University several STR databases were established for several cities Baghdad, Anbar, Najaf, Diyala. The aim of this work is to establish STR database for Hilla population and investigate its parameters.

\section{Materials and Methods}

Samples: Sixty six buccal swabs were collected from healthy unrelated individuals lived in Hilla City were used for analysis.

DNA extraction: Prepfiller (Applied Biosystem, USA, Catalog number: 4463351) was used for DNA purification according to company instructions.

DNA quantification: Quantifiler Real time PCR was used for DNA quantification (Applied Biosystem, USA, Catalog number: 4343895) according to company instructions.

STR loci amplification: Identifiler kit (Applied Biosystem, USA, Catalog number: 4322288) was used for amplification of fifteen autosomal STR loci in tested individuals according to company instructions.

Genetic analysis: Amplified STR loci were analyzed by Genetic Analyzer 3130X1 (Applied Biosystem, USA) according to company instructions.

Allele frequency and Forensic parameters: PowerStat excel software (Promega) was used for analysis of allele frequency and forensic parameters (Matching Probability, Power of Discrimination, Polymorphism Information Content, Power of Exclusion, Paternity Index, Homozygosity, Heterozygosity, and HardyWeinberg equilibrium.

Hardy Wienberg: Arlquin Software (8) was used for analysis of Hardy Wienberg equilibrium. 


\section{Results}

Sixty six volunteers where chosen randomly from Hilla city. Buccal swabs were taken from volunteers for DNA extraction, quantification, PCR amplification and analysis. All samples analyzed successfully with full STR profiles for 15 autosomal and $\mathrm{X}, \mathrm{Y}$ loci. Allele frequency estimated as shown in Table (1).

Table (1a): Allele frequencies of 15 STR loci Hilla city population

\begin{tabular}{|c|c|c|c|c|c|c|c|c|c|}
\hline $\begin{array}{c}\text { Allel } \\
\mathbf{e}\end{array}$ & D8S1179 & D21S11 & D7S820 & $\begin{array}{c}\text { CSF1P } \\
0\end{array}$ & D3S1358 & TH01 & D13S317 & $\begin{array}{c}\text { D16S53 } \\
9 \\
\end{array}$ & Allele \\
\hline 5 & & & & & & & & & 5 \\
\hline 6 & & & & & & 0.25 & & 0.008 & 6 \\
\hline 7 & & & & & 0.008 & 0.152 & & & 7 \\
\hline 8 & & & 0.189 & & & 0.152 & 0.152 & 0.053 & 8 \\
\hline 9 & 0.008 & & 0.129 & 0.03 & & 0.258 & 0.045 & 0.167 & 9 \\
\hline 9.3 & & & & & & 0.167 & & & 9.3 \\
\hline 10 & 0.098 & & 0.28 & 0.258 & & 0.015 & 0.098 & 0.098 & 10 \\
\hline 11 & 0.061 & & 0.273 & 0.295 & & & 0.25 & 0.25 & 11 \\
\hline 12 & 0.053 & & 0.129 & 0.386 & & & 0.348 & 0.288 & 12 \\
\hline 12.2 & & & & & & & & & 12.2 \\
\hline 13 & 0.25 & & & 0.015 & 0.015 & & 0.076 & 0.106 & 13 \\
\hline 13.2 & & & & & & & & & 13.2 \\
\hline 14 & 0.212 & & & 0.008 & 0.076 & & 0.008 & 0.008 & 14 \\
\hline 14.2 & & & & & & & & & 14.2 \\
\hline 15 & 0.22 & & & & 0.265 & & 0.008 & & 15 \\
\hline 15.2 & & & & & & & & & 15.2 \\
\hline 16 & 0.09 & & & 0.008 & 0.288 & 0.008 & 0.008 & & 16 \\
\hline 16.2 & & & & & & & & & 16.2 \\
\hline 17 & 0.008 & & & & 0.227 & & 0.008 & & 17 \\
\hline 17.2 & & & & & & & & & 17.2 \\
\hline 18 & & & & & 0.106 & & & & 18 \\
\hline 18.2 & & & & & & & & & 18.2 \\
\hline 19 & & & & & 0.015 & & & 0.008 & 19 \\
\hline 20 & & & & & & & & & 20 \\
\hline 21 & & & & & & & & & 21 \\
\hline 21.2 & & & & & & & & & 21.2 \\
\hline 22 & & & & & & & & & 22 \\
\hline 22.2 & & & & & & & & & 22.2 \\
\hline 23 & & & & & & & & & 23 \\
\hline 23.2 & & & & & & & & & 23.2 \\
\hline 24 & & & & & & & & & 24 \\
\hline 24.2 & & & & & & & & & 24.2 \\
\hline 25 & & & & & & & & 0.008 & 25 \\
\hline 26 & & & & & & & & 0.008 & 26 \\
\hline 27 & & 0.008 & & & & & & & 27 \\
\hline 28 & & 0.159 & & & & & & & 28 \\
\hline 29 & & 0.265 & & & & & & & 29 \\
\hline 29.2 & & & & & & & & & 29.2 \\
\hline
\end{tabular}




\begin{tabular}{|c|c|c|c|c|c|c|c|c|c|}
\hline 30 & & $\mathbf{0 . 1 8 9}$ & & & & & & & 30 \\
\hline 30.2 & & 0.023 & & & & & & & 30.2 \\
\hline 31 & & 0.061 & & & & & & & 31 \\
\hline 31.2 & & 0.083 & & & & & & & 31.2 \\
\hline 32 & & & & & & & & & 32 \\
\hline 32.2 & & 0.144 & & & & & & & 32.2 \\
\hline 33 & & 0.008 & & & & & & & 33 \\
\hline 33.2 & & 0.061 & & & & & & & 33.2 \\
\hline 34 & & & & & & & & & 34 \\
\hline 34.2 & & & & & & & & & 34.2 \\
\hline 35 & & & & & & & & & 35 \\
\hline$N$ & 66 & 66 & 66 & 66 & 66 & 66 & 66 & 66 & $N$ \\
\hline
\end{tabular}

Table 1a continued:

\begin{tabular}{|c|c|c|c|c|c|c|c|c|}
\hline Allele & D2S1338 & D19S433 & VWA & TPOX & D18S51 & D5S818 & FGA & Allele \\
\hline 6 & & & & & & & & 6 \\
\hline 7 & & & & & & & & 7 \\
\hline 8 & 0.008 & & 0.015 & 0.492 & & & & 8 \\
\hline 9 & & & & 0.106 & & 0.091 & & 9 \\
\hline 9.3 & & & & & & & & 9.3 \\
\hline 10 & 0.008 & 0.008 & & 0.091 & & 0.136 & & 10 \\
\hline 11 & & 0.023 & 0.008 & 0.25 & 0.015 & 0.311 & & 11 \\
\hline 12 & 0.008 & 0.114 & 0.008 & 0.03 & 0.159 & 0.326 & & 12 \\
\hline 12.2 & & & & & & & & 12.2 \\
\hline 13 & 0.015 & 0.227 & & & 0.091 & 0.121 & & 13 \\
\hline 13.2 & & 0.023 & & & & & & 13.2 \\
\hline 14 & 0.008 & 0.25 & 0.045 & & 0.227 & 0.015 & & 14 \\
\hline 14.2 & & 0.076 & & 0.008 & & & & 14.2 \\
\hline 15 & 0.008 & 0.136 & 0.053 & 0.008 & 0.152 & & & 15 \\
\hline 15.2 & & 0.061 & & & & & & 15.2 \\
\hline 16 & 0.03 & 0.03 & 0.212 & & 0.106 & & 0.008 & 16 \\
\hline 16.2 & & 0.038 & & & & & & 16.2 \\
\hline 17 & 0.227 & 0.008 & 0.439 & & 0.106 & & & 17 \\
\hline 17.2 & & & & & & & & 17.2 \\
\hline 18 & 0.152 & 0.008 & 0.129 & 0.008 & 0.083 & & 0.008 & 18 \\
\hline 18.2 & & & & & & & & 18.2 \\
\hline 19 & 0.083 & & 0.083 & & 0.03 & & 0.054 & 19 \\
\hline 20 & 0.114 & & 0.008 & 0.008 & 0.015 & & 0.038 & 20 \\
\hline 21 & 0.038 & & & & 0.015 & & 0.20 & 21 \\
\hline 21.2 & & & & & & & 0.023 & 21.2 \\
\hline 22 & 0.03 & & & & & & 0.138 & 22 \\
\hline 22.2 & & & & & & & & 22.2 \\
\hline 23 & 0.114 & & & & & & 0.154 & 23 \\
\hline 23.2 & & & & & & & 0.008 & 23.2 \\
\hline
\end{tabular}




\begin{tabular}{|c|c|l|l|l|l|l|c|c|}
\hline 24 & 0.083 & & & & & & 0.162 & 24 \\
\hline 24.2 & & & & & & & & 24.2 \\
\hline 25 & 0.068 & & & & & & 0.115 & 25 \\
\hline 26 & 0.008 & & & & & & 0.085 & 26 \\
\hline 27 & & & & & & & 0.008 & 27 \\
\hline 28 & & & & & & & & 28 \\
\hline 29 & & & & & & & & 29 \\
\hline 29.2 & & & & & & & & 29.2 \\
\hline 30 & & & & & & & & 30 \\
\hline$N$ & 66 & 66 & 66 & 66 & 66 & 66 & 66 & $N$ \\
\hline
\end{tabular}

Alleles frequencies were used for determination some forensic, paternity and population genetic parameters as shown in Table (2).

Table (2a): Forensic statistical parameters for each of 15 autosomal STR loci in 66 Hilla city individuals

\begin{tabular}{|l|c|c|c|c|c|c|c|c|}
\hline Parameters & D8S1179 & D21S11 & D7S820 & CSF1PO & D3S1358 & TH01 & D13S317 & D16S539 \\
\hline $\begin{array}{l}\text { Matching } \\
\text { Probability }\end{array}$ & $\mathbf{0 . 0 6 8}$ & $\mathbf{0 . 0 6 1}$ & $\mathbf{0 . 1 0 9}$ & $\mathbf{0 . 1 4 4}$ & $\mathbf{0 . 0 9 9}$ & $\mathbf{0 . 1 0 0}$ & $\mathbf{0 . 0 9 1}$ & $\mathbf{0 . 0 9 6}$ \\
\hline $\begin{array}{l}\text { Power of } \\
\text { Discrimination }\end{array}$ & $\mathbf{0 . 9 3 2}$ & $\mathbf{0 . 9 3 9}$ & $\mathbf{0 . 8 9 1}$ & $\mathbf{0 . 8 5 6}$ & $\mathbf{0 . 9 0 1}$ & $\mathbf{0 . 9 0 0}$ & $\mathbf{0 . 9 0 9}$ & $\mathbf{0 . 9 0 4}$ \\
\hline $\begin{array}{l}\text { Polymorphism } \\
\text { Information } \\
\text { Content }\end{array}$ & $\mathbf{0 . 8 0}$ & $\mathbf{0 . 8 1}$ & $\mathbf{0 . 7 4}$ & $\mathbf{0 . 6 4}$ & $\mathbf{0 . 7 4}$ & $\mathbf{0 . 7 7}$ & $\mathbf{0 . 7 4}$ & $\mathbf{0 . 7 8}$ \\
\hline $\begin{array}{l}\text { Power of } \\
\text { Exclusion }\end{array}$ & $\mathbf{0 . 6 6 2}$ & $\mathbf{0 . 6 3 3}$ & $\mathbf{0 . 6 0 5}$ & $\mathbf{0 . 2 6 3}$ & $\mathbf{0 . 6 9 2}$ & $\mathbf{0 . 5 7 7}$ & $\mathbf{0 . 6 3 3}$ & $\mathbf{0 . 6 0 5}$ \\
\hline Paternity Index & $\mathbf{3 . 0 0}$ & $\mathbf{2 . 7 5}$ & $\mathbf{2 . 5 4}$ & $\mathbf{1 . 1 8}$ & $\mathbf{3 . 3 0}$ & $\mathbf{2 . 3 6}$ & $\mathbf{2 . 7 5}$ & $\mathbf{2 . 5 4}$ \\
\hline Homozygosity & $\mathbf{1 6 . 7 \%}$ & $\mathbf{1 8 . 2 \%}$ & $\mathbf{1 9 . 7 \%}$ & $\mathbf{4 2 . 4 \%}$ & $\mathbf{1 5 . 2 \%}$ & $\mathbf{2 1 . 2 \%}$ & $\mathbf{1 8 . 2 \%}$ & $\mathbf{1 9 . 7 \%}$ \\
\hline Heterozygosity & $\mathbf{8 3 . 3 \%}$ & $\mathbf{8 1 . 8 \%}$ & $\mathbf{8 0 . 3 \%}$ & $\mathbf{5 7 . 6 \%}$ & $\mathbf{8 4 . 8 \%}$ & $\mathbf{7 8 . 8 \%}$ & $\mathbf{8 1 . 8 \%}$ & $\mathbf{8 0 . 3 \%}$ \\
\hline $\begin{array}{l}\text { Hardy- } \\
\text { Weinberg (p- } \\
\text { value) }\end{array}$ & $\mathbf{0 . 3 7 9 0 2}$ & $\mathbf{0 . 1 4 6 5 1}$ & $\mathbf{0 . 1 0 7 1 5}$ & $\mathbf{0 . 1 9 9 4 8}$ & $\mathbf{0 . 8 3 3 6 4}$ & $\mathbf{0 . 0 0 9 4 6}$ & $\mathbf{0 . 2 9 9 4 9}$ & $\mathbf{0 . 0 0 0 3 6}$ \\
\hline $\begin{array}{l}\text { Total Alleles } \\
\text { Observed }\end{array}$ & $\mathbf{1 3 2}$ & $\mathbf{1 3 2}$ & $\mathbf{1 3 2}$ & $\mathbf{1 3 2}$ & $\mathbf{1 3 2}$ & $\mathbf{1 3 2}$ & $\mathbf{1 3 2}$ & $\mathbf{1 3 2}$ \\
\hline
\end{tabular}

Table (2b): Forensic statistical parameters for each of 15 autosomal STR loci in 66 Hilla city individuals

\begin{tabular}{|l|l|l|l|l|l|l|l|}
\hline Parameters & D2S1338 & D19S433 & vWA & TPOX & D18S51 & D5S818 & FGA \\
\hline $\begin{array}{l}\text { Matching } \\
\text { Probability }\end{array}$ & $\mathbf{0 . 0 4 2}$ & $\mathbf{0 . 0 6 0}$ & $\mathbf{0 . 1 1 0}$ & $\mathbf{0 . 1 6 7}$ & $\mathbf{0 . 0 4 5}$ & $\mathbf{0 . 1 0 4}$ & $\mathbf{0 . 0 4 7}$ \\
\hline $\begin{array}{l}\text { Power of } \\
\text { Discrimination }\end{array}$ & $\mathbf{0 . 9 5 8}$ & $\mathbf{0 . 9 4 0}$ & $\mathbf{0 . 8 9 0}$ & $\mathbf{0 . 8 3 3}$ & $\mathbf{0 . 9 5 5}$ & $\mathbf{0 . 8 9 6}$ & $\mathbf{0 . 9 5 3}$ \\
\hline $\begin{array}{l}\text { Polymorphism } \\
\text { Information } \\
\text { Content }\end{array}$ & $\mathbf{0 . 8 7}$ & $\mathbf{0 . 8 2}$ & $\mathbf{0 . 7 0}$ & $\mathbf{0 . 6 3}$ & $\mathbf{0 . 8 5}$ & $\mathbf{0 . 7 2}$ & $\mathbf{0 . 8 5}$ \\
\hline $\begin{array}{l}\text { Power of } \\
\text { Exclusion }\end{array}$ & $\mathbf{0 . 7 5 2}$ & $\mathbf{0 . 6 0 5}$ & $\mathbf{0 . 4 9 7}$ & $\mathbf{0 . 4 4 7}$ & $\mathbf{0 . 4 9 7}$ & $\mathbf{0 . 4 9 7}$ & $\mathbf{0 . 7 4 9}$ \\
\hline Paternity Index & $\mathbf{4 . 1 3}$ & $\mathbf{2 . 5 4}$ & 1.94 & 1.74 & 1.94 & 1.94 & 4.06 \\
\hline Homozygosity & $12.1 \%$ & $\mathbf{1 9 . 7 \%}$ & $\mathbf{2 5 . 8 \%}$ & $\mathbf{2 8 . 8 \%}$ & $\mathbf{2 5 . 8 \%}$ & $\mathbf{2 5 . 8 \%}$ & $\mathbf{1 2 . 3 \%}$ \\
\hline Heterozygosity & $\mathbf{8 7 . 9 \%}$ & $\mathbf{8 0 . 3 \%}$ & $\mathbf{7 4 . 2 \%}$ & $\mathbf{7 1 . 2 \%}$ & $\mathbf{7 4 . 2 \%}$ & $\mathbf{7 4 . 2 \%}$ & $\mathbf{8 7 . 7 \%}$ \\
\hline $\begin{array}{l}\text { Hardy-Weinberg } \\
\text { (p-value) }\end{array}$ & $\mathbf{0 . 0 6 2 8 2}$ & $\mathbf{0 . 0 6 1 9 9}$ & $\mathbf{0 . 4 9 6 8 6}$ & $\mathbf{0 . 0 5 3 8 5}$ & $\mathbf{0 . 0 3 7 9 1}$ & $\mathbf{0 . 3 9 2 4 7}$ & $\mathbf{0 . 1 7 3 5 5}$ \\
\hline $\begin{array}{l}\text { Total Alleles } \\
\text { Observed }\end{array}$ & 132 & 132 & 132 & 132 & 132 & 132 & 132 \\
\hline
\end{tabular}


Table (3) shows that the most common alleles in Hilla city is the same as previously studied for Iraqi population (9).

Table (3): The three most common alleles at each locus for this study of 66 Hilla city individuals and a previous study of southern and central Iraqis (9)

\begin{tabular}{|c|c|c|}
\hline & $\begin{array}{l}\text { Alleles with frequencies from this } \\
\text { study of Baghdad Iraqi Muslims }\end{array}$ & $\begin{array}{l}\text { Allele with frequencies from previous } \\
\text { study }\end{array}$ \\
\hline Locus & $\#$ sample $=132$ alleles & \# sample $=206$ alleles \\
\hline D8S1179 & $13(0.25), 14(0.212), 15(0.22)$ & $15(0.218), 13(0.213), 14(0.140)$ \\
\hline D21S11 & $29(0.265), 30(0.189), 32.2(0.144)$ & $30(0.242), 29(0.222), 32.2(0.148)$ \\
\hline D7S820 & $8(0.189), 10(0.28), 11(0.273)$ & $10(0.331), 11(0.207), 8(0.188)$ \\
\hline CSF1PO & $10(0.258), 11(0.295), 12(0.386)$ & $12(0.328), 11(0.284), 10(0.264)$ \\
\hline D3S1358 & $14(0.076), 15(0.265), 16(0.288)$ & $17(0.364), 16(0.237), 15(0.228)$ \\
\hline TH01 & $6(0.25), 9(0.258), 7(0.152)$ & $6(0.305), 9(0.237), 7(0.169)$ \\
\hline D13S317 & $12(0.348), 11(0.25), 8(0.152)$ & $12(0.344), 8(0.233), 11(0.223)$ \\
\hline D16S539 & $12(0.288), 11(0.25), 8(0.167)$ & $11(0.354), 12(0.291), 9(0.135)$ \\
\hline D2S1338 & $\begin{array}{l}17(0.227), 18(0.152), 20(0.114), 23 \\
(0.114)\end{array}$ & $17(0.223), 20(0.179), 23(0.131)$ \\
\hline D19S433 & $13(0.227), 14(0.25), 15(0.136)$ & $14(0.228), 15(0.218), 13(0.184)$ \\
\hline vWA & $17(0.439), 16(0.212), 18(0.129)$ & $16(0.320), 18(0.228), 17(0.218)$ \\
\hline TPOX & $\begin{array}{l}8(0.492), 11(0.25), 9(0.106), 10 \\
(0.091)\end{array}$ & $8(0.543), 11(0.242), 10(0.106)$ \\
\hline D18S51 & $\begin{array}{l}14(0.227), 12(0.159), 15(0.152), 13 \\
(0.091)\end{array}$ & $14(0.201), 13(0.176), 12(0.147)$ \\
\hline D5S818 & $\begin{array}{l}12(0.326), 11(0.311), 10(0.136), 13 \\
(0.121)\end{array}$ & $12(0.349), 11(0.320), 13(0.203)$ \\
\hline FGA & $21(0.20), 23(0.154), 24(0.162)$ & $24(0.208), 23(0.199), 21(0.165)$ \\
\hline
\end{tabular}

\section{Discussion}

In this study allele frequency of 15 autosomal loci for Hilla city was successfully established and analyzed. STR database can be used for forensic, paternity and criminal investigation. This database adds more information for Iraqi allele's distribution across the country and in comparison with other countries. Forensic parameters (MP, PD, and PIC) were within optimum values for using this database for forensic application. Paternity parameters (PI and PE) were within optimum values for paternity tests analysis.

The main limitation of this study is the number of samples, since international studies suggests that the total number of samples should be more than 150 but due to limited resources, we used 66 samples. P values of WH test should be smaller than 0.05 for all loci to indicate that the population is stable and volunteers chosen randomly and they are not related. Deviation from WHE may be due to the lower number of samples or due to the relatedness among people in Hilla city as in most Iraqi cities. The most common allele's frequencies in this study are almost the same as the study achieved by international researchers for Iraqi in middle and east of Iraq (9) which give additional support to our results.

Additional studies are recommended using high number of samples selected randomly and investigate their relatedness carefully. In addition, comparison of Hilla city database with other cities databases in Iraq, when they completed, to address the genetic variability across the country. Also comparison with other Arabic countries also indicates their genetic relatedness.

It could conclude that this STR database can be used for forensic and paternity applications in Hilla city. 


\section{References}

1. Abu Halima MS, Bernal LP, Sharif FA .Genetic variation of 15 autosomal short tandem repeat (STR) loci in the Palestinian population of Gaza Strip. Leg Med (Tokyo). (2009); 11: 203-204.

2. Picornell A, Toma`s C, Castron JA, Ramon MM. STR data for 13 loci from Jewish populations. International Congress Series 1239. (2003): 109- 112.

3. Barni F, Berti A, Pianese A, Boccellino A, Miller MP, Caperna A, Lago G. Allele frequencies of 15 autosomal STR loci in the Iraq population with comparisons to other populations from the middle-eastern region. Forensic Sci Int. (2007) Mar 22; 167(1): 87-92.

4. Souiden Y, Chaieb K, Chehab O, Mahdouani K. Allele frequencies of ten short tandem repeats loci in the central Tunisian human population. J Genet. (2009) Apr; 88(1): 119-122.

5. Projić P, Škaro V, Šamija I, Pojskić N, Durmić-Pašić A, Kovačević L, Bakal N, Primorac D, Marjanović D. Allele frequencies for 15 short tandem repeat locus in representative sample of croatian population. Croat Med J. (2007); 48: 473-477.

6. Noris G, Santana C, Meraz-Ríos MA, et al. Mexican mestizo population sub-structure: effects on genetic and forensic statistical parameters. Molecular Biology Reports. (2012); 39(12): 10139-10156.

7. Goodwin W, Linacre A, Hadi S. An introduction to forensic genetics. John Wiley \& Sons Ltd. (2007)

8. Excoffier L, Lischer HEL. Arlequin suite ver 3.5: A new series of programs to perform population genetics analyses under Linux and Windows. Molecular Ecology Resources. (2010); 10: 564-567.

9. Barni F, Berti A, Pianese A, Boccellino A, Miller MP, Caperna A, et al. Allele frequencies of 15 autosomal STR loci in the Iraq population with comparisons to other populations from the Middle-Eastern region. Forensic Sci Int. Elsevier. (2007); 167(1): 87-92. 